\title{
A SECOND-ORDER ROSENBROCK METHOD APPLIED TO PHOTOCHEMICAL DISPERSION PROBLEMS*
}

\author{
J. G. VERWER ${ }^{\dagger}$, E. J. SPEE ${ }^{\dagger}$, J. G. BLOM ${ }^{\dagger}$, AND W. HUNDSDORFER ${ }^{\dagger}$
}

\begin{abstract}
A second-order, L-stable Rosenbrock method from the field of stiff ordinary differential equations is studied for application to atmospheric dispersion problems describing photochemistry, advective, and turbulent diffusive transport. Partial differential equation problems of this type occur in the field of air pollution modeling. The focal point of the paper is to examine the Rosenbrock method for reliable and efficient use as an atmospheric chemical kinetics box-model solver within Strang-type operator splitting. In addition, two W-method versions of the Rosenbrock method are discussed. These versions use an inexact Jacobian matrix and are meant to provide alternatives for Strang-splitting. Another alternative for Strang-splitting is a technique based on so-called source-splitting. This technique is briefly discussed.
\end{abstract}

AMS subject classifications. Primary, 65M06, 65M20; Secondary, 65Y05, 65Y20

Key words. long range transport air pollution models, numerical methods, time-dependent advection-diffusion reaction, stiff ODEs, Rosenbrock methods, splitting, approximate factorization

\section{PII. S1064827597326651}

1. Introduction. Photochemical dispersion models are used to enhance the understanding of the chemical composition of the atmosphere, in particular with regard to the relation between anthropogenic emissions and the resulting distributions of primary and secondary polluting species. Modern models are based on mass balances in the form of systems of time-dependent, three-space dimensional, partial differential equations (PDEs) describing advective transport, turbulent diffusive transport, cumulus cloud convection, chemical reactions, emissions, and depositions. Models are discretized on Eulerian grids over areas of different sizes, from urban to regional to fully global. Comprehensive dispersion models are computationally extremely expensive. After spatial discretization they lead to huge systems of ordinary differential equations (ODEs) containing stiff nonlinear terms from the photochemical reactions [32]. For the time integration of these ODEs one commonly uses Strang-type operator splitting [15,23], distinguishing mainly between transport and chemistry. The chemistry computation then amounts to repeatedly solving box-models $\dot{c}=f(c)$ at any of the spatial grid points. For this purpose dedicated explicit methods are used $[8,11,27,30]$ or solvers from the stiff ODE field adjusted for exploiting sparsity in the Jacobian matrix $[16,10,18,17,29]$. As a rule, black-box solvers are considered too costly.

The accuracy demand for atmospheric dispersion problems is modest, so a loworder method is a natural choice. Rosenbrock methods have already proven very effective for low to modest accuracies for a wide variety of stiff problems [7]. When sparsity is exploited, their specific advantage for integrating atmospheric box-models has recently been demonstrated in [17], where the stiffly accurate solver RODAS3

* Received by the editors August 27, 1997; accepted for publication (in revised form) April 6, 1998; published electronically March 30, 1999. The research of the first author was supported by Cray Research Inc. via National Computing Facilities Foundation grant CRG 97.02. The research of the second author was supported by RIVM-the National Institute of Public Health and Environmental Protection project CIRK. The research of the third and fourth authors was supported by the Dutch HPCN program project TASC, Task $1 \mathrm{~A}$

http://www.siam.org/journals/sisc/20-4/32665.html

†CWI, P.O. Box 94079, 1090 GB Amsterdam, The Netherlands (jan.verwer@cwi.nl, edwin. spee@knmi.nl, joke.blom@cwi.nl, w.h.hundsdorfer@cwi.nl). 
came out as most competitive. This solver is a variable step size, third-order, fourstage counterpart of the well-known fourth-order, six-stage solver RODAS from [7]. However, like most solvers, RODAS3 is quite sensitive to initial transients and therefore can require a rather small step size in the initial phase to start up the integration. For single ODE systems and long integration intervals, a small initial step renders no problem, as it hardly adds to the total work load. In the context of operator splitting the situation is different. The integration intervals are relatively short and usually small transients are encountered within any split step. These transients are insignificant, and for efficiency reasons it is desirable to start up with an a priori described step size far greater than the smallest time constant. In addition, when running operator splitting schemes on vector/parallel or massively parallel computers, a priori described step sizes everywhere seem more practical than truly variable ones. Variable step size integration governed by local error control creates load imbalancing in parallel implementations [6] and interferes with vectorization over the horizontal grid dimension $[10,21]$.

These observations have led us to search for a more stable Rosenbrock formula which is capable of integrating with much larger a priori described step sizes. The result is a particular, L-stable, second-order, two-stage method. In what follows, this method will be called ROS2 (Rosenbrock, second order). The main difference between ROS2 and RODAS3 lies in the stability function and the internal stability functions. For ROS2 both these functions are positive along the negative real line, like the exponential. This does not hold for RODAS3. In this paper we provide strong numerical evidence that this simple positivity property significantly enhances the nonlinear stability for atmospheric chemical kinetic problems, thus rendering ROS2 highly efficient for use within Strang-type operator splitting. Here lies the focal point of this paper. Another difference with RODAS3 is that for second-order consistency, ROS2 can be used with an inexact Jacobian matrix. This property can be exploited in different ways and we pay some attention to it in connection with alternatives for standard Strang-splitting. We note in passing that Rosenbrock methods using an inexact Jacobian are also called W-methods [7].

The contents of the paper are as follows. In section 2 we outline the main intended application for ROS2 through a prototype model for three-dimensional (3D) spherical photochemical dispersion. This prototype model will be used as a test problem along with three different sets of atmospheric photochemical reactions from the actual practice. In section 3 we present ROS2, discuss the basic properties which render this Rosenbrock method suitable and highly competitive as an atmospheric box-model integrator, and provide numerical results to illustrate this. In section 4 we apply ROS2 within the context of Strang-splitting: Splitting chemistry is decoupled from transport, yielding a potential difficulty for the chemistry integration in that stiff transients can arise in any split step. These transients are artifacts of the splitting and require a robust and stable method. The main objective of this section is to show that ROS2 is well capable for this task. Two different second-order Strang-splittings are used. The first treats advection and diffusion explicit and coupled, the second treats advection explicit and vertical diffusion implicit but still coupled. At this stage we introduce a W-version of ROS2 as an advection-diffusion solver. We use this solver within Strang-splitting, but it is of obvious interest of its own. Section 5 is devoted to a complete alternative for Strang-splitting. Here we present a second application of ROS2 as a W-method, but now for the full advection-diffusion-chemistry problem. The approximate factorization approach is used here for the inexact Jacobian defini- 
tion. This approximate factorization ROS2 scheme has been introduced to provide a comparison with Strang-splitting for cases where there is large vertical turbulent diffusion. Standard Strang-splitting then yields larger splitting errors and comparison with alternatives is of numerical interest. The result of the present comparison is that the approximate factorization ROS2 scheme certainly is competitive, but since Strang-splitting is somewhat simpler for use in the actual practice, greater benefits must be shown to replace it. In section 6 we briefly discuss another alternative for Strang-splitting, which is called source-splitting. However, on theoretical grounds it is argued that Strang-splitting is more stable. The main conclusion of our investigations is formulated in the final section, section 7 .

2. Photochemical dispersion models. By way of illustration we first outline the spherical, global prototype model from [21], which later will serve as a test model. Let $c=c(t, \lambda, \phi, r)$ denote a vector of $m$ species concentrations with $t$ representing the time, $\lambda \in[0,2 \pi]$ the longitude, $\phi \in\left[-\frac{\pi}{2},+\frac{\pi}{2}\right]$ the latitude, and $0 \leq r \leq r_{H}$ the height above the surface of the earth. Let $a$ be the radius of the earth, $\rho$ the density of the air, $(u, v)$ a horizontal wind field, and $K$ a vertical subgrid-scale turbulent diffusion coefficient. Let $f(c)$ be an $m$-dimensional vector function representing atmospheric (photo)chemical reactions, emission sources, and sink depositions. The mathematical formulation of the prototype model then reads

$$
\frac{\partial c}{\partial t}+\frac{1}{a \cos \phi}\left[\frac{\partial(u c)}{\partial \lambda}+\frac{\partial(v c \cos \phi)}{\partial \phi}\right]=\frac{\partial}{\partial r}\left(\rho K \frac{\partial}{\partial r}\left(\frac{c}{\rho}\right)\right)+f(c) .
$$

The reaction term $f(c)$ couples the $m$ equations in the model. Without reactions the equations are not coupled, since $u, v, \rho$, and $K$ are given expressions. Processes are time and space dependent. In real models the various meteorological parameters are updated every few hours, say. For numerical purposes we consider all processes constant in time over split intervals. At the surface and the top the no-flux boundary conditions

$$
\rho K \frac{\partial}{\partial r}\left(\frac{c}{\rho}\right)=0
$$

are imposed. The model is completed by prescribing the initial values for $c$ at the initial time $t=t_{0}$. The unit for the concentrations is number of molecules per $\mathrm{cm}^{3}$ $\left(\mathrm{mlc} / \mathrm{cm}^{3}\right)$. The unit of time is seconds.

2.1. Transport. In the prototype model the wind field $(u, v)$ and the coefficient $K$ are given analytic expressions. The wind field is a solid body rotation [20] with a maximum speed of about $125 \mathrm{~km} /$ hour defined by

$$
u=2 \pi \kappa(\cos \beta \cos \phi+\sin \beta \sin \phi \cos \lambda), \quad v=-2 \pi \kappa \sin \beta \sin \lambda,
$$

where $\beta=45^{\circ}$ and $\kappa=a /(14 * 24 * 3600)$ with $a$ equal to $6378 \mathrm{~km}$. The diffusion coefficient $K$ is dependent on the height and takes on a maximum of $30 \mathrm{~m}^{2} / \mathrm{s}$ in the lower troposphere. Adopting a parameterization given in [31, p. 24, Figs. 1-10], we used $\left(K\right.$ in $\mathrm{m}^{2} / \mathrm{s}, z$ in $\left.\mathrm{km}\right)$

$$
\begin{array}{ll}
K=30 & \text { if } z \leq 15 \\
K=0.2 & \text { if } 15<z \leq 17.5 \\
K=0.2+0.32(z-17.5) & \text { if } 17.5<z \leq 20 \\
K=10^{0.05 z-1.0} & \text { if } z>20
\end{array}
$$


The top of the model lies at $34.7 \mathrm{~km}$. The density $\rho$ is height dependent and chosen in close accordance with the U.S. Standard Atmosphere (1976) [31]. Its definition is in tabulated form, which is too long to specify. Real models also simulate transport by vertical advection, subgrid-scale turbulent horizontal diffusion, and subgrid-scale cumulus cloud convection. For a numerical study horizontal diffusion is not really essential. Horizontal diffusion can always be added and numerically integrated explicitly, in a similar manner as horizontal advection. In a similiar manner, vertical advection can often be treated explicitly. If not, it can be combined somehow with the implicit vertical diffusion computation. Whether the absence of cloud convection is essential is as yet not clear. In reality there is also orography. This makes models technically much more complicated, as this amounts to a coordinate transformation of the ideal sphere model (2.1). We believe, however, that with regard to time stepping the absence of orography is not essential either.

2.2. Chemistry. Atmospheric photochemistry induces severe stiffness. Reaction times may range from milliseconds or shorter (e.g., OH radical) to years (e.g., $\mathrm{CH}_{4}$ ). The photochemical nature complicates the numerical solution, since part of the reaction coefficients depends on the solar zenith angle, which depends on the time of the day and the location on earth. This dependence gives rise to constantly moving areas of rapid solution change coupled to sunset and sunrise. There also exists a dependence on the temperature and the pressure. This dependence is chosen in close accordance with the U.S. Standard Atmosphere (1976) [31]. In applications the number of species varies, between 20 and 100, say. In numerical illustrations presented later, we use three different sets of chemical reactions, all borrowed from the actual practice:

- Chemistry model RIVM. The first set consists of 45 reactions between $m=17$ species and is used in actual long-term global studies, where it is referred to as methane chemistry. See the appendix of the preprint of [29].

- Chemistry model CBM-IV. The second set is based on the carbon bond mechanism IV consisting of $m=32$ chemical species involved in 70 thermal and 11 photolytic reactions. We also used this model in [17], but with a different solar zenith angle. To stress the numerical method, we have prescribed high emission values (the urban scenario from [17]).

- Chemistry model WET. The third set contains $m=65$ species involved in 77 thermal and 11 photolytic gas-phase chemical reactions, 39 liquid-phase chemical reactions, and 39 gas-liquid mass transfer reactions. The gas-phase mechanism is based on the carbon bond mechanism IV, while the liquid-phase mechanism is based on a chemical scheme from [14]. We also used this model in [17], again with a different solar zenith angle, and we emphasize that it is the most difficult one from the three mentioned here due to the heterogeneous reactions.

2.3. Spatial discretization. Model (2.1) is discretized on a 3D Eulerian grid spanning the entire globe. The longitude-latitude grid is uniform, except near the poles, where the grid is reduced (coarsened) in the longitude direction to alleviate the CFL restriction for explicit advection schemes caused by the pole singularity. The spatial advection scheme is based on a mass-conservative, cell-centered, flux-limited, third-order upwind discretization. Flux limiting is used for positivity. See [9] and [21] for details.

The spatial vertical diffusion scheme is based on cell-centered three-point finite differences. The vertical grid is nonuniform. The prototype model has 15 layers. The 
distribution of the cell vertical centers is a function of the pressure which is taken uniform over the globe. The lowest cell boundary lies at sea level $(1000 \mathrm{hPa})$ and the highest at $38.2 \mathrm{~km}(0 \mathrm{hPa})$. The complete distribution of the cell centers reads 0.3 , $1.0,2.2,4.3,6.5,8.4,10.0,11.3,13.0,15.2,17.6,19.8,22.5,27.6,34.7 \mathrm{~km}$.

In [21] three different reduced longitude-latitude grids were used. Without grid reduction their dimensions are $64 \times 32,128 \times 64$, and $256 \times 128$, respectively. With grid reduction this leads to 1656,6264 , and 26104 horizontal grid points, so that combined with the vertical grid totals of 24840,93960 , and 391560 grid points were used. Recall that for any grid point and any of the $m$ species, a nonlinear ODE must be integrated in time, revealing the enormous computational scale of atmospheric dispersion modeling and the necessity of developing highly efficient, tailored algorithms. See also the recent monograph of Zlatev [32].

3. Solving box-models. In atmospheric dispersion modeling one frequently applies operator splitting and employs stiff ODE solvers to integrate resulting boxmodels. In this section we therefore first consider the box-model

$$
\dot{c}=f(c)
$$

contained in (2.1). We will outline our specific choice of Rosenbrock method, discuss the basic properties which render this method suitable and highly competitive as an atmospheric box-model integrator, and provide numerical results to illustrate this.

3.1. The ROS2 integration formula. Our starting point is a family of nonautonomous two-stage Rosenbrock methods discussed on page 233 in [5]:

$$
\begin{aligned}
c_{n+1} & =c_{n}+\tau b_{1} k_{1}+\tau b_{2} k_{2}, \\
k_{1} & =f\left(t_{n}, c_{n}\right)+\tau \gamma A k_{1}, \\
k_{2} & =f\left(t_{n}+\alpha_{21} \tau, c_{n}+\tau \alpha_{21} k_{1}\right)+\tau \gamma_{21} A k_{1}+\tau \gamma A k_{2},
\end{aligned}
$$

where $c_{n} \approx c(t)$ at $t=t_{n}, \tau=t_{n+1}-t_{n}$ is the step size, and $A$ is the Jacobian matrix $f^{\prime}\left(t_{n}, c_{n}\right)$ or an approximation thereof. The method is second-order consistent for any $A$ iff

$$
b_{1}=1-b_{2}, \quad \gamma_{21}=-\gamma / b_{2}, \quad \alpha_{21}=1 /\left(2 b_{2}\right)
$$

with $\gamma$ and $b_{2} \neq 0$ still free. We will use the autonomous form, and in this section we assume that $A=f^{\prime}\left(c_{n}\right)$, which ensures conservation of mass. The parameter $\gamma$ appears in the stability function

$$
R(z)=\frac{1+(1-2 \gamma) z+\left(\frac{1}{2}-2 \gamma+\gamma^{2}\right) z^{2}}{(1-\gamma z)^{2}} .
$$

This function is A-stable iff $\gamma \geq 1 / 4$. Since atmospheric chemistry models contain radicals with a very short life span we want L-stability, that is, $R(\infty)=0$. This is achieved by $\gamma=1 \pm 1 / \sqrt{2}$. Further, we select $b_{2}=\frac{1}{2}$. Avoiding the matrix-vector multiplication in the second stage computation, and at the same time redefining $k_{2}$ by $k_{2}-2 k_{1}$, we then rewrite the resulting autonomous ROS2 scheme in the form

$$
\begin{aligned}
c_{n+1} & =c_{n}+\frac{3}{2} \tau k_{1}+\frac{1}{2} \tau k_{2}, \\
(I-\gamma \tau A) k_{1} & =f\left(c_{n}\right), \\
(I-\gamma \tau A) k_{2} & =f\left(c_{n}+\tau k_{1}\right)-2 k_{1} .
\end{aligned}
$$


Observe that the intermediate approximation $c_{n}+\tau k_{1}$ is first-order consistent at $t=t_{n+1}$ and hence can be used to provide a cheap local error estimation for step size control. In the present investigation we will not exploit this possibility since we focus on using a priori described step sizes as mentioned in the introduction.

3.2. Stability and positivity. For $\gamma$ we select the larger value $\gamma_{+}=1+1 / \sqrt{2}$ in spite of the fact that this gives a larger error coefficient in the leading local truncation error. Numerical tests have revealed that this yields a notably better nonlinear stability behavior for large step sizes. A balanced explanation fails, but we conjecture that the following linear property plays a role. If $\gamma=\gamma_{+}$, then $R(z)$ is positive for all real negative $z$, whereas this is not true for the smaller value $\gamma_{-}=1-1 / \sqrt{2}$. Positivity of $R$ presumably has some advantage for nonlinear chemical kinetic systems

$$
\dot{c_{k}}=f_{k}(c) \equiv P_{k}(c)-L_{k}(c) c_{k},
$$

where $P_{k}(c)$ contains all production terms for the $k$ th species and $L_{k}(c) c_{k}$ represents the losses for this species. Suppose that for a certain species, $P_{k}$ and $L_{k}$ are truly constant. Then,

$$
c_{k, n+1}=R(z) c_{k, n}+\frac{R(z)-1}{z} \tau P_{k}, \quad z=-\tau L_{k} \leq 0 .
$$

If $R(z)<0$, then $c_{k, n+1}$ might become negative. On the other hand, if $0 \leq R(z) \leq 1$, then $c_{k, n+1} \geq 0$ is guaranteed. This obviously proves nothing for truly nonlinear systems. However, in the atmosphere the so-called radicals react very fast and are always near to their steady state value $P_{k}(c) / L_{k}(c)$. If the dependence of $P_{k}, L_{k}$ on $c$ is very weak, the above linear reasoning can come close to what happens in the Rosenbrock computation. Even a very small negative solution value can cause sign problems, because radicals occur in a muitiplication with very large positive reaction constants. Another advantage of $\gamma_{+}$is that the internal stability function

$$
R_{1}(z)=\frac{1+(1-\gamma) z}{1-\gamma z}
$$

associated with the first-stage approximation $c_{n}+\tau k_{1}$ also satisfies $0<R_{1}(z)<1$ for all real negative $z$, something which does not hold for $\gamma_{-}$. This property of internal stability has already been shown to be of practical interest for nonlinear stiff ODE problems in [26].

Of further interest is that $\gamma_{+}$is also to be preferred when solving the nonlinear scalar model problem

$$
\dot{c}=\lambda c^{2}, \quad \lambda<0,
$$

whose solution

$$
c(t)=\frac{c(0)}{1-\lambda c(0) t}
$$

remains positive if $c(0)$ is positive. This simple model nicely reveals the danger of a negative solution value, since the scalar Jacobian $2 \lambda c$ is positive if $c$ is negative. In this situation the problem can thus become severely unstable and its solution may not even exist. 
TABLE 1

Sparsity data for the three sets of chemical reactions.

\begin{tabular}{||l||c|c|c||}
\hline Chemical reaction set & WET & CBM-IV & RIVM \\
Number of entries in $F^{\prime}$ & $65^{2}$ & $32^{2}$ & $17^{2}$ \\
Number of nonzeros in $F^{\prime}$ & 506 & 276 & 100 \\
Number of nonzeros after sparse LU & 629 & 300 & 107 \\
\hline
\end{tabular}

Denote $z=\tau \lambda$. The approximations $c_{n}+\tau k_{1}$ and $c_{n+1}$ then read

$$
c_{n}+\tau k_{1}=\frac{c_{n}+(1-2 \gamma) z c_{n}^{2}}{1-2 \gamma z c_{n}}
$$

and

$$
c_{n+1}=\frac{c_{n}+(1-6 \gamma) z c_{n}^{2}+\left(1-6 \gamma+12 \gamma^{2}\right) z^{2} c_{n}^{3}+\left(\frac{1}{2}-2 \gamma+8 \gamma^{2}-8 \gamma^{3}\right) z^{3} c_{n}^{4}}{\left(1-2 \gamma z c_{n}\right)^{3}} .
$$

It easily follows that both are unconditionally positive for $\gamma=\gamma_{+}$, whereas both can become negative if $\gamma=\gamma_{-}$. For the intermediate approximation $c_{n}+\tau k_{1}$, the term $(1-2 \gamma) z c_{n}^{2}$ in the numerator fails to be unconditionally positive and for $c_{n+1}$ this is the case for the term $\left(\frac{1}{2}-2 \gamma+8 \gamma^{2}-8 \gamma^{3}\right) z^{3} c_{n}^{4}$.

3.3. Clipping. For real photochemical systems positivity cannot be guaranteed. Because it is essential for stability, in the application of ROS2 positivity is enforced at both the stages by clipping. This means that when a component of $c_{n}+\tau k_{1}$ or $c_{n+1}$ is negative, it is set equal to zero. Clipping interferes with the property of mass conservation. However, in our experiments we have not observed a notable loss in accuracy, presumably because in an actual integration ROS2 is clipping only occasionally if $\gamma=\gamma_{+}$. In section 3.5 we will provide numerical evidence for this observation. Lest we miss the obvious, enforcing positivity by clipping does not guarantee stability.

3.4. Workload and sparsity. Each time step with ROS2 requires an evaluation of the Jacobian $A=f^{\prime}\left(c_{n}\right)$, two linear system solutions accompanied with two derivative evaluations. The Jacobian update and the solution of the linear systems, requiring one matrix factorization (LU-decomposition) and two backsolves (forwardbackward substitutions), account for most of the CPU time. Fortunately, for large atmospheric chemistry models the number of zeros in the Jacobian is substantial. For very large models it readily amounts to $\approx 90 \%$. This high level of sparsity can be exploited to significantly reduce the costs of these linear algebra calculations. For this purpose we use the symbolic preprocessor $\operatorname{KPP}[4,16]$ in the same way as in [17]. KPP takes as input a set of chemical reactions and delivers the production and loss terms defining the ODE system $\dot{c}=f(c)$. Most important is that it also prepares a sparse matrix factorization with only a minimal fill-in and that it delivers a routine for the backsolve without indirect addressing. All together this means that the numerical algebra can be handled very efficiently, leading to a substantial reduction of the workload for large chemical kinetic models. Table 1 shows sparsity data for the three models WET, CBM-IV, and RIVM.

3.5. Numerical illustrations. Numerical results will be shown for three boxmodels based on the reaction sets RIVM, CBM-IV, and WET. Starting from an arbitrary initial state far from chemical equilibrium, in all tests we simulate that we 


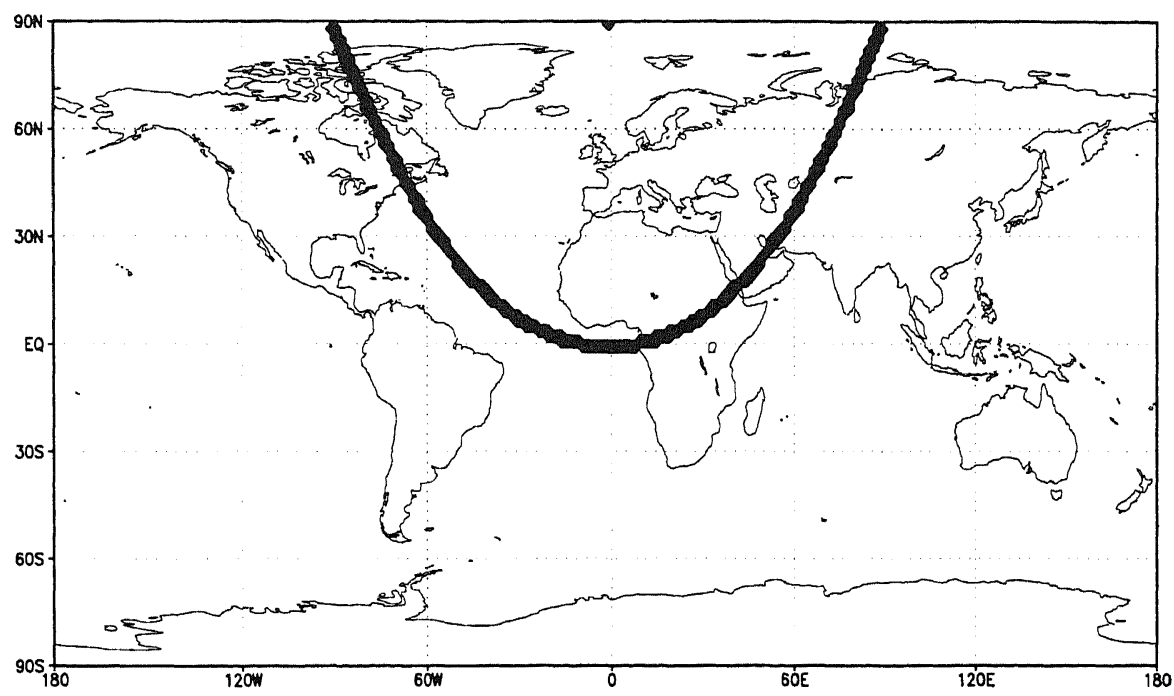

FIG. 1. The trajectory used in the box-model tests and one-dimensional tests.

follow an air parcel from its release point east of Africa for 14 days along the trajectory shown in Figure 1, picking up emissions along the way. After 14 days the air parcel returns at its release point. Because to a great extent the initial conditions are chosen arbitrarily, the first day is used as start-up time (integration over the first day is carried out nearly exactly using a very small step size). It should be noted that the trajectory passes the North Pole at day 7 , in the neighborhood of which the photochemical reactions are weaker than elsewhere. This effect leads to a disturbance in the diurnal behavior which can be observed in the species solutions. Hourly frozen reaction coefficients were used with an update halfway between each hour interval. This renders the ODE systems autonomous and implies that the pressure, temperature, and solar angle are taken piecewise constant rather than time-continuous. In actual practice one normally operates this way, one reason being that many of the coefficients are expensive to compute.

We first present results of a stability test, comparing ROS2 for $\gamma=\gamma_{+}$and $\gamma=\gamma_{-}$. We have also included the related Rosenbrock method RODAS3 proposed in [17] in this test. RODAS3 is based on a stiffly accurate, embedded pair of order $3(2)$. It has four stages and uses three function evaluations. In our stability test we have used only the third-order formula, which is given by

$$
\begin{aligned}
c_{n+1} & =c_{n}+\frac{5}{6} \tau k_{1}-\frac{1}{6} \tau k_{2}-\frac{1}{6} \tau k_{3}+\frac{1}{2} \tau k_{4}, \\
\left(I-\frac{1}{2} \tau A\right) k_{1} & =f\left(c_{n}\right) \\
\left(I-\frac{1}{2} \tau A\right) k_{2} & =f\left(c_{n}\right)+A k_{1} \\
\left(I-\frac{1}{2} \tau A\right) k_{3} & =f\left(c_{n}+\tau k_{1}\right)-\frac{1}{4} A k_{1}-\frac{1}{4} A k_{2}, \\
\left(I-\frac{1}{2} \tau A\right) k_{4} & =f\left(c_{n}+\frac{3}{4} \tau k_{1}-\frac{1}{4} \tau k_{2}+\frac{1}{2} \tau k_{3}\right)+\frac{1}{12} A k_{1}+\frac{1}{12} A k_{2}-\frac{2}{3} A k_{3} .
\end{aligned}
$$

In actual integrations we have experienced that this third-order, four-stage method is less stable than ROS2 when using fixed, large step sizes. As for ROS2 using $\gamma=\gamma_{-}$, 
we conjecture that lack of positivity of its stability function

$$
R(z)=\frac{1-z+\frac{1}{6} z^{3}}{\left(1-\frac{1}{2} z\right)^{4}}
$$

plays a role here. Further, from its three internal stability functions

$$
R_{1}(z)=1, \quad R_{2}(z)=\frac{1+\frac{1}{2} z}{1-\frac{1}{2} z}, \quad R_{3}(z)=\frac{1-\frac{1}{2} z-\frac{1}{4} z^{2}}{\left(1-\frac{1}{2} z\right)^{3}}
$$

both $R_{2}$ and $R_{3}$ fail to be positive. As already mentioned in the introduction, this seems an important difference between RODAS3 and ROS2 using $\gamma=\gamma_{+}$, the latter possessing both a positive stability function and a positive internal stability function along the negative real line.

Table 2 shows maximal step sizes for which integrations were found stable. The integrations cover 13 days and start at day 2 from the chemical equilibrium as outlined above. The table gives clear evidence of the superior stability of ROS2 for the larger $\gamma$. Starting from the arbitrary initial states of day 1 far away from the chemical equilibrium shows even greater differences. See the italic numbers in Table 2. The table also reveals that the result of clipping may lead to a smaller step size rather than to a larger one, as happens for $\operatorname{ROS} 2\left(\gamma_{+}\right)$applied to RIVM. However, in all other cases clipping indeed does allow larger step sizes. Of further interest is that, with respect to stability, RODAS3 performs notably better than $\operatorname{ROS} 2\left(\gamma_{-}\right)$but worse than $\operatorname{ROS} 2\left(\gamma_{+}\right)$. However, the difference between the latter two is much less. We believe that RODAS3 owes this to its higher order of consistency, which results in more accuracy. More accuracy will eventually lead to positive solutions and hence to a more stable process.

For this specific test an integration has been called stable if during the whole integration period a certain relative error remains below 10. The precise error definition is not so important, nor is the threshold 10. We used the error expression

$$
\frac{1}{m} \sum_{k=1}^{m} E R_{k}
$$

where

$$
E R_{k}=\sqrt{\frac{1}{\left|\mathcal{J}_{k}\right|} \cdot \sum_{n \in \mathcal{J}_{k}}\left|\frac{c_{k}\left(t_{n}\right)-\hat{c}_{k}\left(t_{n}\right)}{\hat{c}_{k}\left(t_{n}\right)}\right|^{2}}
$$

and

$$
\mathcal{J}_{k}=\left\{0 \leq n \leq N: \hat{c}_{k}\left(t_{n}\right) \geq a_{k}\right\}, \quad a_{k}=\frac{10^{-4}}{N} \sum_{i=1}^{N} \hat{c}_{k}\left(t_{i}\right)
$$

The solution values $\hat{c}_{k}\left(t_{n}\right)$ represent accurate reference solutions at every full hour value $t_{n}$, computed in high precision with the third-order code RODAS3. Recall that $m$ equals the number of species. For the $k$ th species, $E R_{k}$ is a temporal error for which the set $\mathcal{J}_{k}$ has been introduced to remove very small solution values in the relative measurement. This means that $a_{k}$ acts as a threshold which serves to eliminate very small values in the relative error measurement. The integer $N$ equals here $14 * 24$, being the number of output times taken into consideration. 
TABLE 2

Box-model stability test. The numbers are step sizes in seconds with and without clipping. Italic numbers belong to the start point outside chemical equilibrium.

\begin{tabular}{||l||c|c|c||}
\hline Method & WET & CBM-IV & RIVM \\
\hline ROS2 $\left(\gamma_{+}\right)$ & $3600-3600$ & $3600-1800$ & $1200-1800$ \\
& $3600-1800$ & $3600-150$ & $1200-1800$ \\
ROS2 $(\gamma-)$ & $150-90$ & $212-156$ & $400-200$ \\
& $<5-<5$ & $133-36$ & $400-133$ \\
RODAS3 & $1800-400$ & $1800-514$ & $1200-900$ \\
& $1800-<5$ & $1800-133$ & $1200-900$ \\
\hline
\end{tabular}

We proceed with presenting results obtained for the actual box-model integrations by ROS2 and TWOSTEP. The latter is a two-step BDF solver using nonlinear Gauss-Seidel iteration instead of modified Newton for the nonlinear BDF relations [27]. The use of Gauss-Seidel iteration renders this solver effectively explicit and hence cheap. TWOSTEP is capable of solving gas-phase chemistry more efficiently than other dedicated explicit solvers, e.g., QSSA [11,18]. But like all other explicit solvers it cannot handle heterogeneous reactions as in WET. Therefore, results for TWOSTEP only concern the models RIVM and CBM-IV (see also [17]).

For the two species $\mathrm{O}_{3}$ and $\mathrm{HNO}_{3}$, Figures 2 and 3 show concentrations in $\mathrm{mlc} / \mathrm{cm}^{3}$ versus time in hours for day 2 up to day 14 . The figures contain a highly accurate reference solution and the two computed solutions using a fixed step size of $10 \mathrm{~min}$. and $20 \mathrm{~min}$., respectively. Such fixed step sizes are very large for atmospheric chemistry integrations. Observe that in many applications advection step sizes are also in this range.

The Rosenbrock method can be seen to perform very satisfactorily. With the smallest step size it computes $\mathrm{O}_{3}$ and $\mathrm{HNO}_{3}$ for WET up to plotting accuracy. For $\tau=$ 20 min., a mild error growth occurs for this model. Applied to CBM-IV and RIVM, the Rosenbrock method delivers excellent results for both step sizes. TWOSTEP also solves CBM-IV accurately with the smallest step size, but generates significant errors for $\tau=20 \mathrm{~min}$. When applied to RIVM this explicit code clearly requires a smaller step size than the Rosenbrock method.

Noteworthy is that the accuracy for TWOSTEP can be improved by spending more Gauss-Seidel iterations. Only a fixed number of 2 iterations has been used here, as in [17]. This makes it very cheap in CPU. The CPU time needed by ROS2 for CBM-IV and RIVM is only about a factor 2 higher, revealing the efficiency of this linearly implicit solver. In part we owe this to the use of KPP and the sparsity. However, the main advantage of ROS2 over TWOSTEP and related dedicated explicit solvers is that it can deal with different kinds of reactions, including heterogeneous ones as in WET.

4. ROS2 within Strang-type operator splitting. The Rosenbrock solver ROS2 is primarily meant for efficient use in standard, Strang-type operator splitting codes. In this section we will illustrate that the solver is capable of this task by showing results for the prototype model from section 2. Our findings obviously also apply to different models. Adopting the method of lines approach, let

$$
\dot{w}=F(w) \equiv F_{T}(w)+F_{R}(w), \quad F_{T}(w) \equiv F_{A}(w)+F_{D}(w)
$$

denote the ODE system that originates from spatial discretization of the photochemical transport model. Hence $w(t)$ stands for a grid function, and the vector function $F_{T}$ 

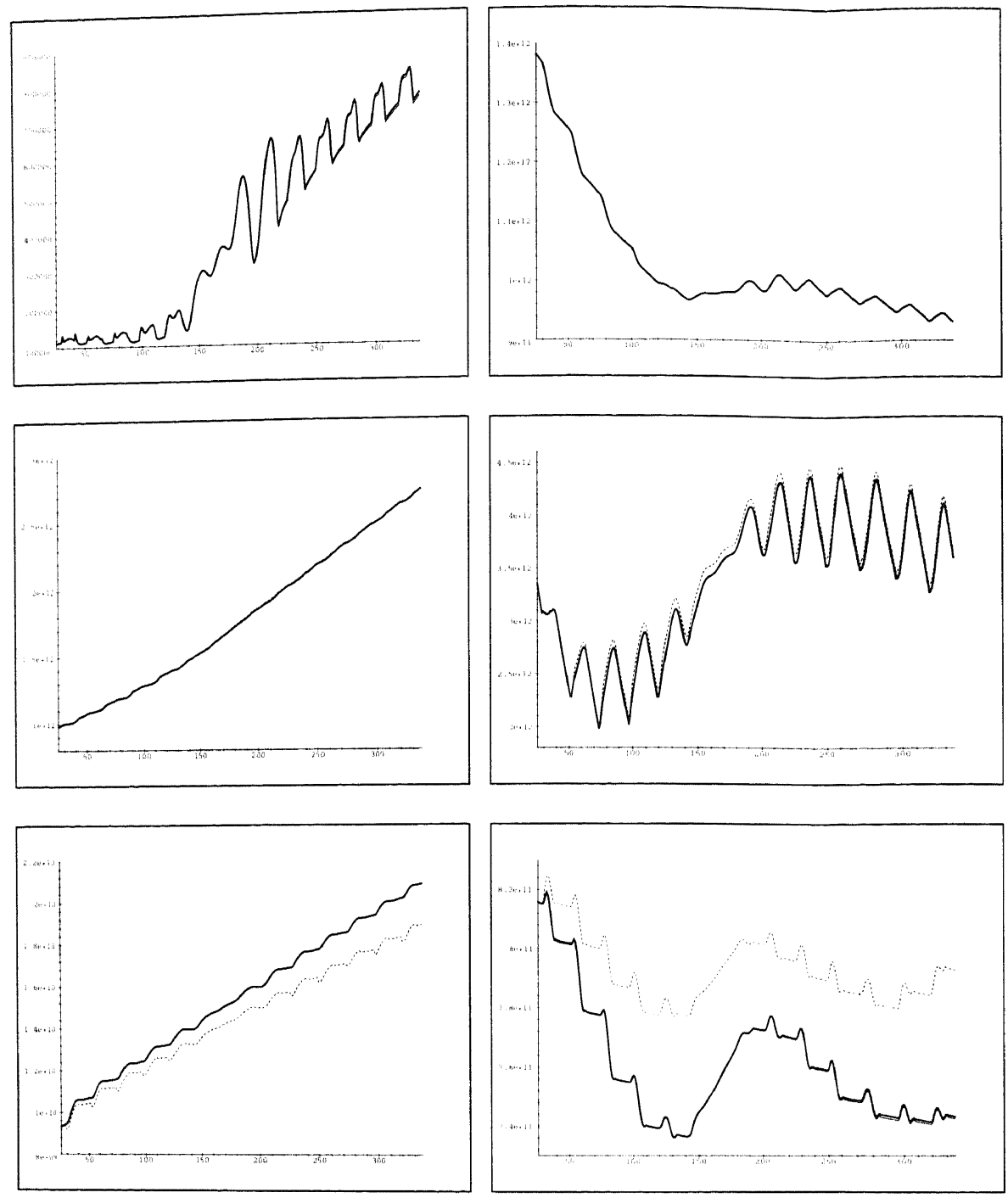

FIG. 2. Box-model test, $\tau=10$ min. Concentrations of $\mathrm{HNO}_{3}$ (left) and $\mathrm{O}_{3}$ (right) in $\mathrm{mlc} / \mathrm{cm}^{3}$ versus time in hours for WET (top), CBM-IV (middle), and RIVM (bottom). Thick solid lines represent the reference solution, thin solid lines the ROS 2 solution, and dotted lines the TWOSTEP solution. TWOSTEP has not been applied to WET.

is supposed to contain the semidiscrete transport contributions from advection and diffusion, represented here by $F_{A}$ and $F_{D}$, respectively. Likewise, $F_{R}$ stems from the chemical reactions, emissions, and depositions. For any grid point the terms in $F_{R}$ are simply the box-model expressions contained in $f$. We will discuss two second-order Strang-splittings, one treating vertical diffusion explicitly and one with an implicit vertical diffusion part.

4.1. Vertical diffusion explicit. For system (4.1) second-order Strang-splitting can be organized in many ways. We consider the combination 

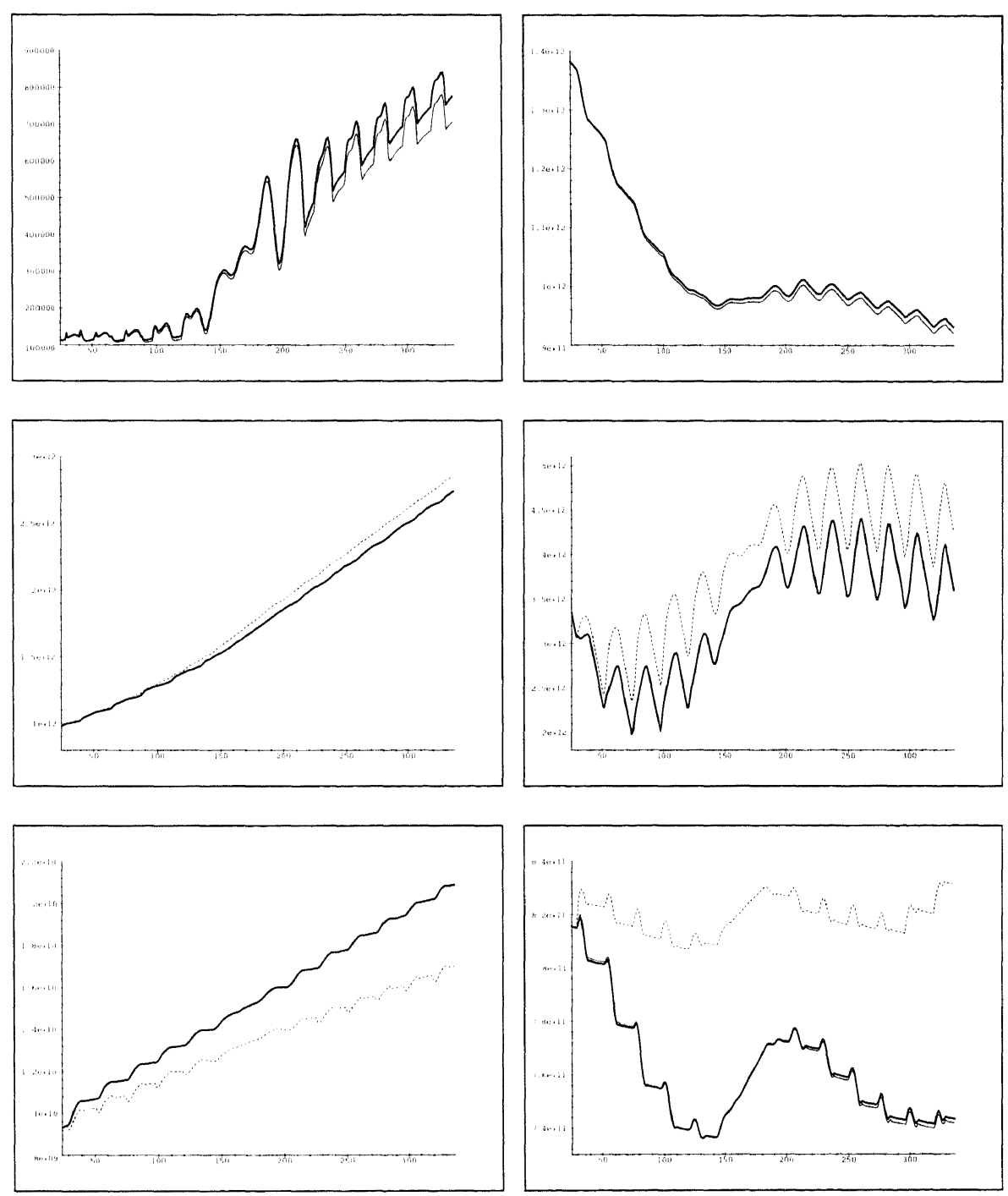

Fig. 3. Box-model test, $\tau=20$ min. Concentrations of $\mathrm{HNO}_{3}$ (left) and $\mathrm{O}_{3}$ (right) in $\mathrm{mlc} / \mathrm{cm}^{3}$ versus time in hours for WET (top), CBM-IV (middle), and RIVM (bottom). Thick solid lines represent the reference solution, thin solid line the ROS2 solution, and dotted lines the TWOSTEP solution. TWOSTEP has not been applied to WET.

$$
\begin{aligned}
W_{0} & =w_{n}, \\
W_{1} & =W_{0}+\frac{1}{4} \tau F_{T}\left(W_{0}\right)+\frac{1}{4} \tau F_{T}\left(W_{0}+\frac{1}{2} \tau F_{T}\left(W_{0}\right)\right), \\
W_{2} & =W_{1}+\frac{3}{2} \tau k_{1}+\frac{1}{2} \tau k_{2}, \\
W_{3} & =W_{2}+\frac{1}{4} \tau F_{T}\left(W_{2}\right)+\frac{1}{4} \tau F_{T}\left(W_{2}+\frac{1}{2} \tau F_{T}\left(W_{2}\right)\right), \\
w_{n+1} & =W_{3},
\end{aligned}
$$

where $k_{1}$ and $k_{2}$ are given by

$$
(I-\gamma \tau A) k_{1}=F_{R}\left(W_{1}\right), \quad(I-\gamma \tau A) k_{2}=F_{R}\left(W_{1}+\tau k_{1}\right)-2 k_{1}
$$


and $A=F_{R}^{\prime}\left(W_{1}\right)$. Hence the reaction part is treated with ROS2 and the transport part by the explicit trapezoidal rule. We note in passing that this rule derives from ROS2 by substitution of the zero matrix for $A$. By standard operator splitting we thus solve transport and chemistry in a sequential, symmetric manner such that the chemistry computation becomes completely decoupled from the transport. An advantage of standard splitting is that it amounts to chemistry box-model computations over the space grid and that it is easy to implement and is memory efficient. A disadvantage is that the decoupling can result in stiff transients within any split step as the decoupled transport changes the solution values for the chemistry integration. These stiff transients are a numerical artifact and may complicate the chemistry integration.

4.1.1. Stability. By using the explicit trapezoidal rule for the advective-diffusive transport, we tacitly assume that this does not lead to severe stability restrictions on the time step. In [9] and [21] stability of the explicit trapezoidal rule has been discussed for pure advection when using third-order upwind discretization with flux limiting. A CFL number of $\frac{2}{3}$ was shown to lead to a stable and positive advection computation. For practical purposes this is quite satisfactory. When vertical diffusion is also included, the following is necessary:

$$
\tau \max \frac{4 K}{(\Delta r)^{2}} \approx 1.0
$$

For our model (2.1) this condition allows sufficiently large step sizes. For example, substitution of the values for $K$ and $\Delta r$ given in section 2 yields $\tau \approx 700^{2} /(4 \times 30) \approx$ 4100 seconds. In cases of much finer vertical meshes or much larger values for $K$, the explicit trapezoidal rule will no longer be efficient and must be replaced to obtain an implicit vertical diffusion computation.

4.1.2. $1 \mathrm{D}$ results. We first show numerical results for the one-dimensional (1D) diffusion-reaction system

$$
\frac{\partial c}{\partial t}=\frac{\partial}{\partial r}\left(\rho K \frac{\partial}{\partial r}\left(\frac{c}{\rho}\right)\right)+f(c), \quad 0 \leq r \leq r_{H},
$$

obtained from (2.1) for zero velocities. These results are of interest in their own as they enable a comparison between the 1D Strang-splitting scheme and ROS2 directly applied to the semidiscrete 1D problem $\dot{w}=F(w) \equiv F_{D}(w)+F_{R}(w)$ using the full Jacobian matrix. Needless to say, in 3D this is not feasible.

The same three chemistry models are used as in the box-model tests, now simulating the evolution of an air column along the trajectory of Figure 1. The initial values for $c$ are chosen such that the mixing ratios $c / \rho$ are independent of $r$, while the initial values at ground level are the same as for the box-models. Step sizes are chosen in the same way as in the box-model tests, i.e., $\tau=10 \mathrm{~min}$. and $\tau=20 \mathrm{~min}$. during day 2 up to day 14 .

We found that for all three chemistry models the 1D ROS2 scheme and the 1D Strang-splitting scheme (4.2) are very close in accuracy. In fact, all computed solutions are in excellent agreement with the reference solution being the semidiscrete solution at ground level computed in high time step accuracy. Figure 4 shows the evolution of $\mathrm{O}_{3}$ and $\mathrm{HNO}_{3}$ along the trajectory at ground level for the reference solution and the two numerical methods using $\tau=20 \mathrm{~min}$. One can see that overall the agreement is almost up to plotting accuracy. This indicates that for the current $1 \mathrm{D}$ problem Strang-splitting has no adverse effect on accuracy whatsoever for step sizes smaller than or equal to $20 \mathrm{~min}$. 

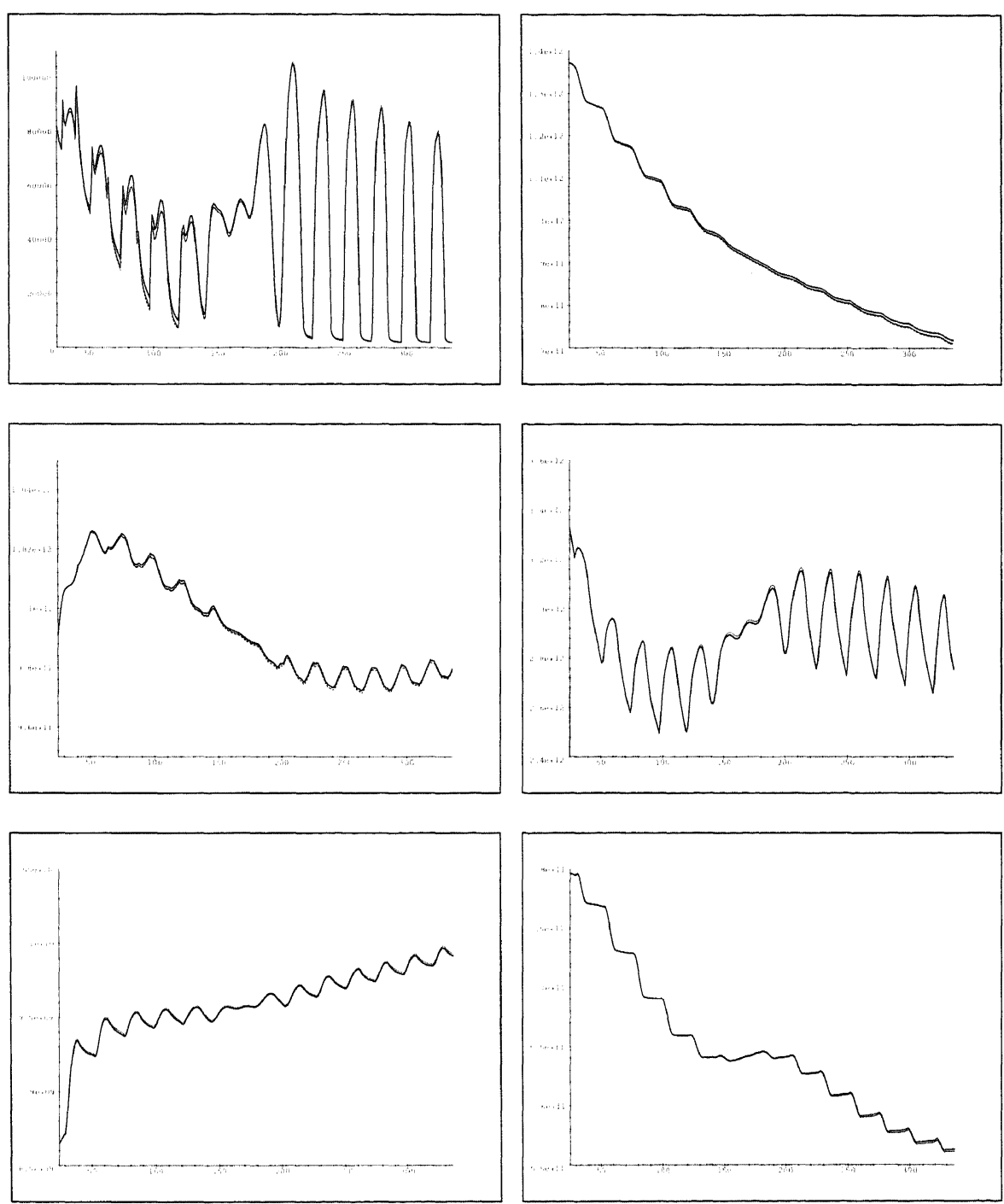

FIG. 4. $1 D$ test, $\tau=20$ min., $K_{\max }=30 \mathrm{~m}^{2} / \mathrm{s}$. Ground level concentrations of $\mathrm{HNO}_{3}$ (left) and $\mathrm{O}_{3}$ (right) in mlc/ $\mathrm{cm}^{3}$ versus time in hours for WET (top), CBM-IV (middle), and RIVM (bottom). Thick solid lines are the reference solution, thin solid lines the Strang-splitting solution (4.2), and dashed lines the $1 D$ ROS2 solution.

It is stressed that when there are a large number of species, the use of the full banded Jacobian in ROS2 is not advocated in real practice, as this involves a considerable numerical algebra overhead (see also [28]). For WET, CBM-IV, and RIVM the Strang-splitting computation is less expensive in CPU time by factors of roughly 19 , 6 , and 4 , respectively.

4.1.3. 3D results. In 3D the Strang-splitting method (4.2) has been applied only for the chemical reaction set RIVM. The test is similar to the one carried out in [21] on the coarsest reduced $64 \times 32$ horizontal space grid, yielding a total of 24840 
grid cells in 3D. The same initial distribution was used. On this grid an accurate, semidiscrete reference solution has been determined to furnish the initial values for the actual integration starting at day 2 , as well as to assess the accuracy at the final time at day 14. We again successfully used fixed step sizes of 10 and 20 min. These step sizes are allowed for the advection computation since on the chosen grid the critical step size for a stable advection computation is about 20 min. Note that in (4.2) the step size $\tau$ is halved in the transport steps.

The results clearly indicate that also in a 3D Strang-splitting code ROS2 is able to integrate the chemical kinetic equations with large step sizes. For an accuracy assessment we refer to Figure 5 . This figure shows ground level profiles of $\mathrm{O}_{3}, \mathrm{HO}_{2} \mathrm{NO}_{2}$, and $\mathrm{HNO}_{3}$, plotted along the horizontal SW-NE grid diagonal of Figure 1 at the final time at day 14. The integers below the horizontal axis refer to the location on the diagonal; 0 points to the $\mathrm{SW}$ corner and 128 to the NE corner. For each species two profiles were plotted, the computed one and the reference profile. Only for $\mathrm{HO}_{2} \mathrm{NO}_{2}$ are the errors notable.

4.2. Vertical diffusion implicit. It is of numerical interest to examine a test case which requires an implicit vertical diffusion computation. For this purpose we have artificially increased the diffusion coefficient $K$ in the prototype model by a factor of 100 so that the maximum value has become 3000 . The explicit trapezoidal rule is then no longer efficient for use in (4.2) and must be replaced. We have replaced it by a new scheme which also keeps the advective and diffusive transport coupled.

The new transport scheme is derived from ROS2 and exploits the fact that this Rosenbrock method is second-order consistent for any choice of the Jacobian approximation $A$. Specifically, we apply ROS2 to the transport problem $\dot{w}=F_{T}(w) \equiv$ $F_{A}(w)+F_{D}(w)$ and choose $A=F_{D}^{\prime}\left(w_{n}\right)$ to obtain

$$
\begin{aligned}
w_{n+1} & =w_{n}+\frac{3}{2} \tau k_{1}+\frac{1}{2} \tau k_{2}, \\
\left(I-\gamma \tau F_{D}^{\prime}\left(w_{n}\right)\right) k_{1} & =F_{T}\left(w_{n}\right), \\
\left(I-\gamma \tau F_{D}^{\prime}\left(w_{n}\right)\right) k_{2} & =F_{T}\left(w_{n}+\tau k_{1}\right)-2 k_{1} .
\end{aligned}
$$

Hence advection is still treated explicitly while, owing to the 1D nature of $F_{D}^{\prime}$, the computation of the stage vectors $k_{1}, k_{2}$ now requires the solution of tridiagonal linear systems, one for each species and each horizontal grid point. With respect to CPU this scheme is therefore almost as cheap per step as the explicit trapezoidal rule. Replacing the explicit trapezoidal rule in (4.2) with (4.5) gives the second-order Strang-splitting scheme

$$
\begin{aligned}
W_{0} & =w_{n}, \\
W_{1} & =W_{0}+\frac{3}{4} \tau k_{1}^{(1)}+\frac{1}{4} \tau k_{2}^{(1)}, \\
W_{2} & =W_{1}+\frac{3}{2} \tau k_{1}^{(2)}+\frac{1}{2} \tau k_{2}^{(2)}, \\
W_{3} & =W_{2}+\frac{3}{4} \tau k_{1}^{(3)}+\frac{1}{4} \tau k_{2}^{(3)}, \\
w_{n+1} & =W_{3},
\end{aligned}
$$

where $W_{1}$ and $W_{3}$ are computed by (4.5) and $W_{2}$ is obtained in the same way as in (4.2).

4.2.1. Stability. Using an approximation for the Jacobian in the Rosenbrock method does of course affect the stability. Following the standard way of reasoning, 

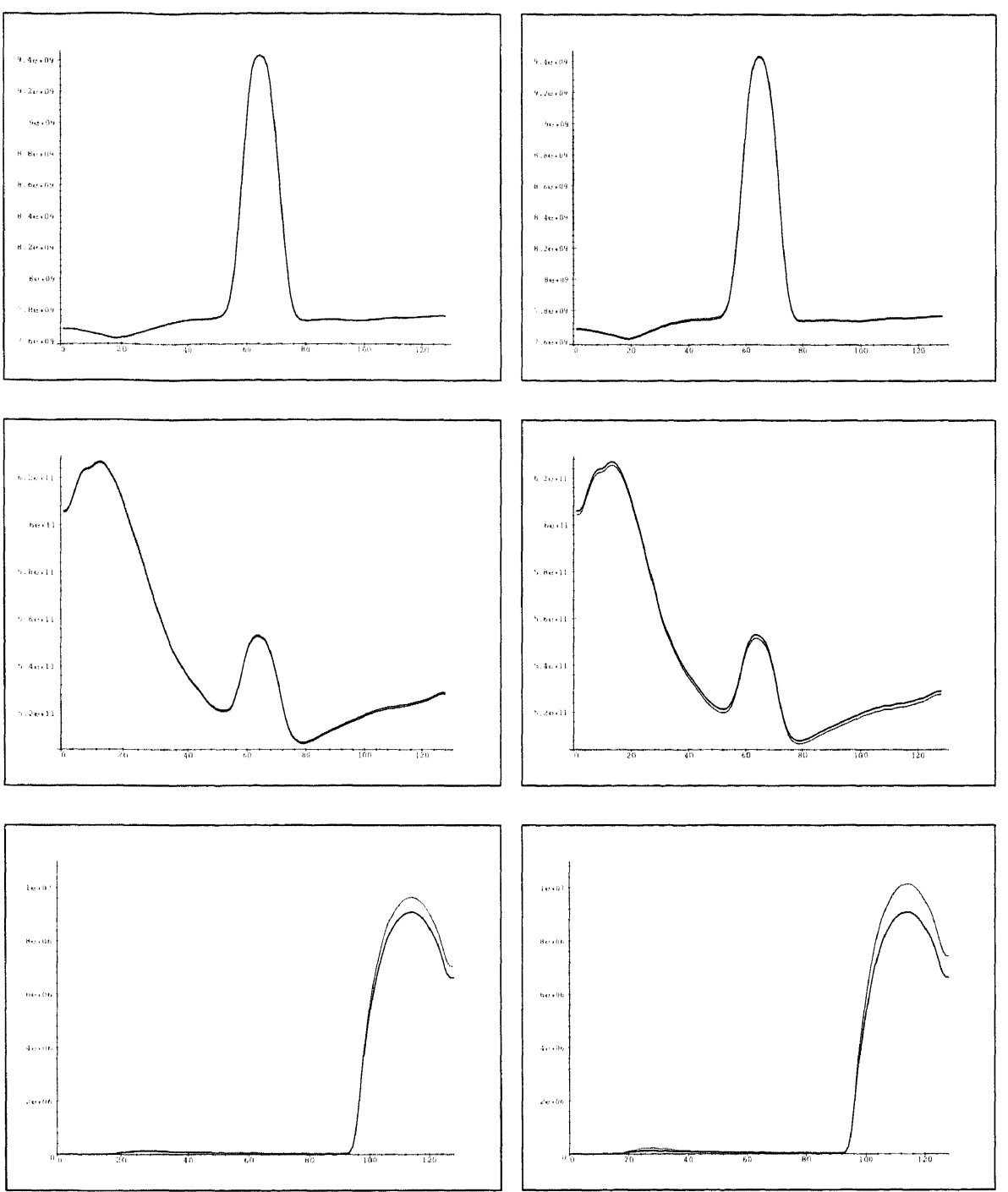

FIG. 5. $3 D$ test, $\tau=10 \min$. (left), $\tau=20 \min$. (right), $K_{\max }=30 \mathrm{~m}^{2} / \mathrm{s}$. Ground level concentrations of $\mathrm{HNO}_{3}$ (top), $\mathrm{O}_{3}$ (middle), and $\mathrm{HO}_{2} \mathrm{NO}_{2}$ (bottom) plotted in mlc/ $\mathrm{cm}^{3}$ along the $S W-N E$ diagonal for the final time at day 14. Thick solid lines are the reference solution and thin solid lines the Strang-splitting solution (4.2).

for the advection-diffusion scheme (4.5) we examine the stability for the scalar, linear test model

$$
\dot{w}=\lambda_{A} w+\lambda_{D} w .
$$

Through an eigenvector-eigenvalue decomposition this scalar model is derived from the constant coefficient linear system

$$
\dot{w}=J_{A} w+J_{D} w
$$


where $J_{A}, J_{D}$ represent "frozen" Jacobians $F_{A}^{\prime}\left(w_{n}\right), F_{D}^{\prime}\left(w_{n}\right)$. Hence, $\lambda_{A}$ represents a complex-valued eigenvalue of the advection Jacobian $F_{A}^{\prime}\left(w_{n}\right)$. Likewise, $\lambda_{D}$ represents a real, nonpositive eigenvalue of the diffusion Jacobian $F_{D}^{\prime}\left(w_{n}\right)$. The eigenvectoreigenvalue decomposition is valid if the matrices share the same eigensystem, in which case it can also be carried through for the Rosenbrock approximations. Assuming spatial discretization on a uniform grid, this decomposition holds for problems with constant coefficients and periodic boundary conditions.

Denote $z_{A}=\tau \lambda_{A}$ and $z_{D}=\tau \lambda_{D}$. The stability function of (4.5) then is

$$
R\left(z_{A}, z_{D}\right)=\frac{\left(1+z_{A}+\frac{1}{2} z_{A}^{2}\right)+(1-2 \gamma)\left(1+z_{A}\right) z_{D}}{\left(1-\gamma z_{D}\right)^{2}}
$$

which reduces to the stability function $R\left(z_{A}, 0\right)=1+z_{A}+\frac{1}{2} z_{A}^{2}$ of the explicit trapezoidal rule for $z_{D}=0$ and to (3.3) for $z_{A}=0$. Because we assume that advection can be computed explicitly, we tacitly assume that $z_{A}=\mathcal{O}(1)$. As a consequence, $R\left(z_{A}, z_{D}\right) \rightarrow 0$ for $z_{D} \rightarrow-\infty$. Thus the damping at infinity property of the original L-stable stability function (3.3) is maintained for the large and negative diffusion eigenvalues.

LEMMA 1. $\left|R\left(z_{A}, z_{D}\right)\right| \leq 1$ whenever $z_{D} \leq 0$ is real and $\left|1+z_{A}+\frac{1}{2} z_{A}^{2}\right| \leq 1$.

Proof. Denote $\theta=(2 \gamma-1) / \gamma= \pm \sqrt{2}$. Using the notation $\tilde{z}=\gamma z_{D}, \alpha=$ $1+z_{A}+\frac{1}{2} z_{A}^{2}$, and $\beta=\theta\left(1+z_{A}\right)$, we can write

$$
R\left(z_{A}, z_{D}\right)=\frac{\alpha-\beta \tilde{z}}{(1-\tilde{z})^{2}}
$$

Suppose $\tilde{z}=-t \leq 0$. Then

$$
\left|R\left(z_{A}, z_{D}\right)\right|^{2}=\frac{|\alpha|^{2}+\eta t+|\beta|^{2} t^{2}}{(1+t)^{4}}, \quad \eta=\alpha \bar{\beta}+\bar{\alpha} \beta=2 \operatorname{Re}(\alpha \bar{\beta}) .
$$

So, if $|\alpha|=1$, we have $\left|R\left(z_{A}, z_{D}\right)\right| \leq 1$ for all $t \geq 0$ iff

$$
\eta+\left|\beta^{2}\right| t \leq 4+6 t+4 t^{2}+t^{3} \quad \text { for all } t \geq 0 \text {. }
$$

Below it will be shown that $|\alpha|=1$ implies

$$
\eta \leq \frac{3}{2} \sqrt{6}<4 \quad \text { and } \quad|\beta|^{2} \leq 6
$$

which proves the lemma. and

To verify the inequalities in (4.9), let $\zeta=1+z_{A}$. Then $\alpha=\frac{1}{2}\left(1+\zeta^{2}\right), \beta=\theta \zeta$,

$$
\eta=\theta\left(1+|\zeta|^{2}\right) \operatorname{Re} \zeta
$$

We consider values of $z_{A}$ such that $|\alpha|=1$. Hence $\left|1+\zeta^{2}\right|=2$, and it easily follows that $|\beta|^{2}=2|\zeta|^{2} \leq 6$. Further, any $\zeta$ satisfying $\left|1+\zeta^{2}\right|=2$ can be parameterized as

$$
\zeta=\cos \phi+i \sqrt{\kappa} \sin \phi, \quad \kappa=1+2|\sin \phi|^{-1} .
$$

By some calculations we thus arrive at $\eta=2 \theta(1+|\sin \phi|) \cos \phi$ and the maximum value for $\eta$ is found to be $\frac{3}{2} \sqrt{6}$.

This lemma proves unconditional stability for all real, nonpositive $z_{D}$ as long as the complex number $z_{A}$ lies in the stability region of the explicit trapezoidal rule. Hence, for the linear model problem the critical step size for stability is equal to the critical step size for advection. The advection-diffusion solver (4.5) is therefore of interest itself and can also prove useful in other applications. 
4.2.2. $1 \mathrm{D}$ results. We have repeated the $1 \mathrm{D}$ test for problem (4.4) with the 100 times larger diffusion coefficient $K$, again comparing the 1D Strang-splitting scheme (4.6) to the 1D ROS2 scheme for $\tau=10,20 \mathrm{~min}$. Figure 6 shows the $\mathrm{O}_{3}$ and $\mathrm{HNO}_{3}$ profiles at ground level in the same manner as in the previous 1D Figure 4 . We see that for all three chemistry models the accuracy of the splitting scheme is still good but has become lower, especially for $\tau=20 \mathrm{~min}$. This is due to the much larger value for $K$. The 1D ROS2 scheme performs equally well and accurately for WET and RIVM. However, it has become unstable for the chemistry model CBM-IV. This happens for both step sizes. This instability is odd in view of the fact that splitting gives a fairly accurate, stable result. For the CBM-IV model the 1D ROS2 plot has been omitted.

4.2.3. 3D results. Figure 7 shows results of the 3D test using the 100 times larger diffusion coefficient $K$. The new Strang-splitting scheme (4.6) can be seen to solve the problem well for both step sizes of 10 and $20 \mathrm{~min}$. (In Figure 7 results are also included for a different method that will be discussed in section 5.) We do encounter larger errors, however, compared with those of the previous 3D figure. The error is very large for $\mathrm{HO}_{2} \mathrm{NO}_{2}$, but for the most important species $\mathrm{O}_{3}$ it remains within acceptable bounds.

It is emphasized that the larger errors are due to the splitting and that the transport and chemistry schemes themselves hardly contribute to the observed errors. In other words, replacing these two schemes by the exact solution operators for the transport problem $\dot{w}=F_{T}(w)$ and the chemistry problem $\dot{w}=F_{R}(w)$, within the framework of Strang-splitting, will not annihilate the error.

The splitting error has also been observed in related 3D tests carried out in [21], where a completely different chemical integrator based on TWOSTEP has been used. Because we artificially increased the expression for $K$ from [31] by a factor of 100 , we must admit that we are not sure whether this $3 \mathrm{D}$ test is really meaningful for the actual practice of computational air quality modeling. On the other hand, it is a sound numerical test which has confirmed the accuracy and robustness of the Rosenbrock method ROS2 as an efficient chemical integrator within Strang-splitting.

5. ROS2 applied with approximate factorization. The main idea behind operator splitting is to avoid the complications of solving the huge systems of linear and nonlinear algebraic equations encountered when applying implicit or linearly implicit time integration methods. Somehow related to splitting is the notion of approximate factorization, where a form of splitting is performed at the numerical algebra level rather than at the operator level. See $[1,2,3,25]$ for examples of approximate factorization. By splitting at the numerical algebra level the operator splitting error is avoided. In this section we will therefore briefly examine whether for our 3D photochemical dispersion problem the Rosenbrock method ROS2 applied with a certain approximate factorization can offer a viable alternative to Strang-splitting for problems with large vertical diffusion. The same approximate factorization approach for the same Rosenbrock method has been proposed independently in [19]. In this thesis no actual results are presented.

As before we consider the ODE system

$$
\dot{w}=F(w) \equiv F_{A}(w)+F_{D}(w)+F_{R}(w)
$$

for which ROS2 reads

$$
w_{n+1}=w_{n}+\frac{3}{2} \tau k_{1}+\frac{1}{2} \tau k_{2},
$$



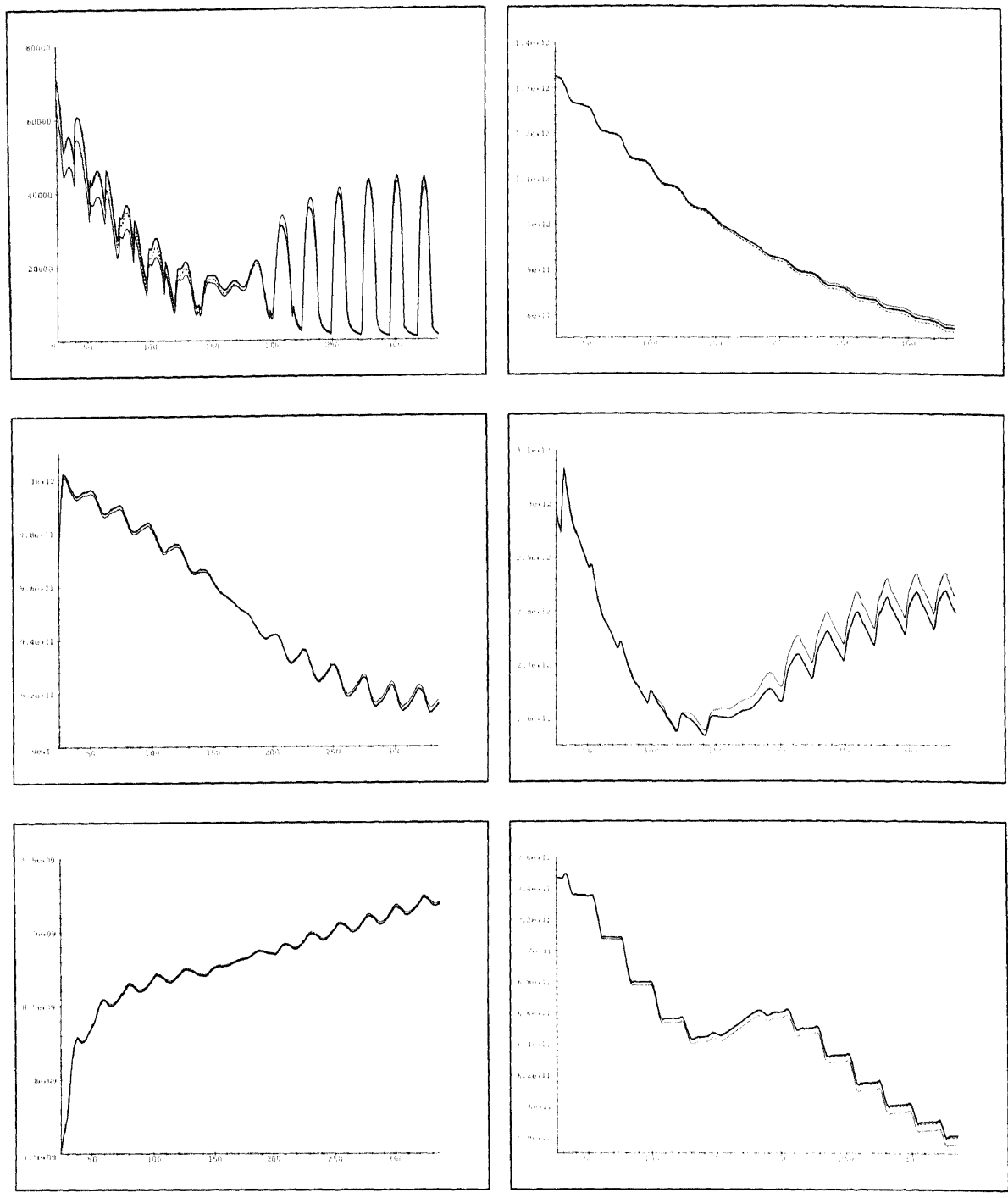

FIG. 6. $1 D$ test, $\tau=20$ min., $K_{\max }=3000 \mathrm{~m}^{2} / \mathrm{s}$. Ground level concentrations of $\mathrm{HNO}_{3}$ (left) and $\mathrm{O}_{3}$ (right) in mlc/cm $\mathrm{cm}^{3}$ versus time in hours for WET (top), CBM-IV (middle), and RIVM (bottom). Thick solid lines are the reference solution, thin solid lines the Strang-splitting solution (4.6), and dashed lines the $1 D$ ROS2 solution.

$$
\begin{aligned}
& (I-\gamma \tau A) k_{1}=F\left(w_{n}\right), \\
& (I-\gamma \tau A) k_{2}=F\left(w_{n}+\tau k_{1}\right)-2 k_{1} .
\end{aligned}
$$

Approximate factorization is applied by defining $A$ such that $I-\gamma \tau A$ is factorized into

$$
I-\gamma \tau A=\left(I-\gamma \tau F_{D}^{\prime}\left(w_{n}\right)\right)\left(I-\gamma \tau F_{R}^{\prime}\left(w_{n}\right)\right) .
$$

The computation of the two stage vectors $k_{1}, k_{2}$ then amounts to a normal $F$-evaluation and two sequential linear system solutions, one for the vertical diffusion and one for 

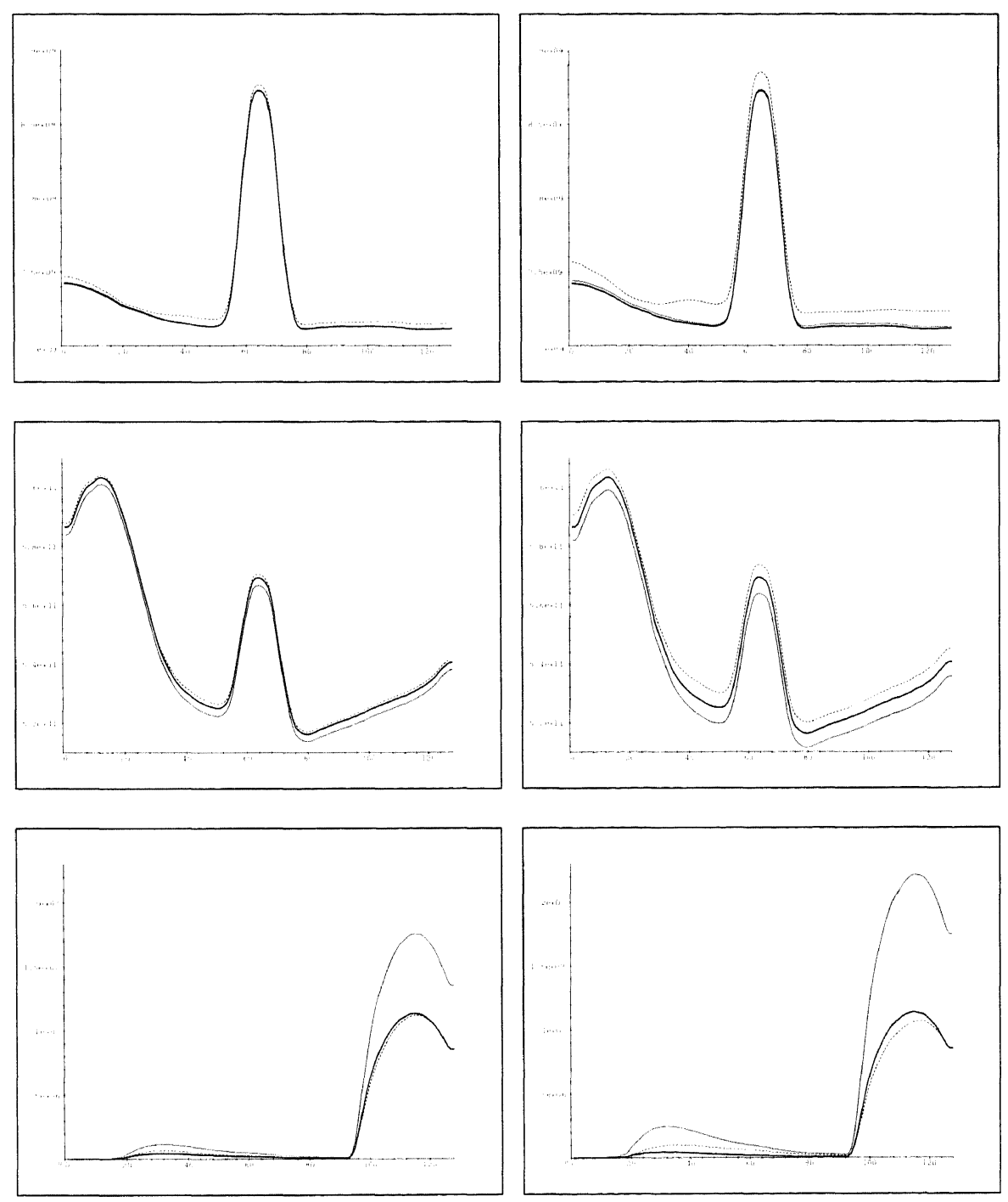

FIG. 7. $3 D$ test, $\tau=10 \mathrm{~min}$. (left), $\tau=20 \mathrm{~min}$. (right), $K_{\max }=3000 \mathrm{~m}^{2} / \mathrm{s}$. Ground level concentrations of $\mathrm{HNO}_{3}$ (top), $\mathrm{O}_{3}$ (middle), and $\mathrm{HO}_{2} \mathrm{NO}_{2}$ (bottom) plotted in mlc/ $\mathrm{cm}^{3}$ along the $S W-N E$ diagonal for the final time at day 14 . Thick solid lines are the reference solution, thin solid lines the Strang-splitting solution (4.6), and dashed lines the factorized ROS2 solution (5.1)-(5.2).

the chemistry. Owing to the box-model structure of $F_{R}^{\prime}$ and the tridiagonal structure of $F_{D}^{\prime}$ (observe the resemblance with (4.5)), these linear systems solutions can be carried out efficiently. The normal $F$-evaluation avoids the decoupling of operator splitting, and the two linear system solutions are introduced for stability reasons. The order in the factorization is important. If we reverse the order, i.e., first solve for the chemistry, the numerical performance will decrease.

5.1. Stability. Similar to (4.5), a separate stability investigation of (5.1) is required. For that purpose we again consider the standard scalar test model, now in the form 

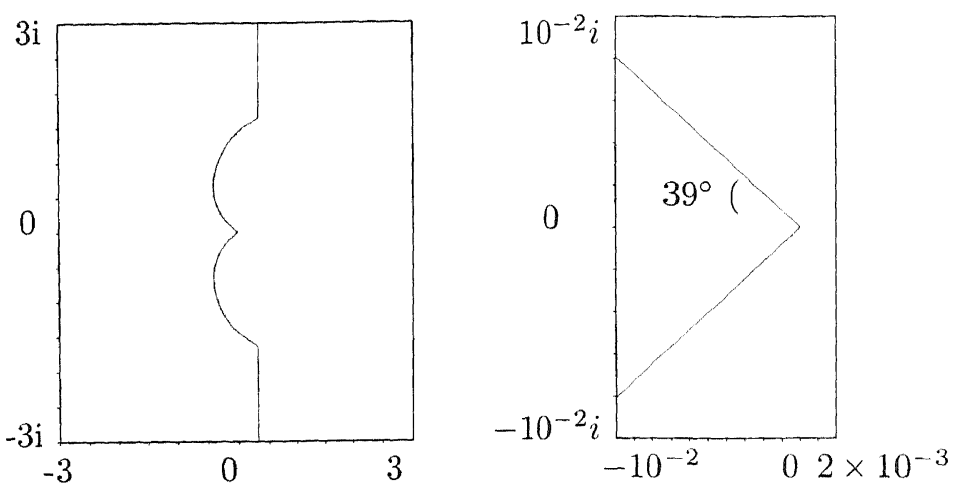

FIG. 8. On the left is the $z_{R}$-stability boundary for the factorized stability function (5.4). On the right is the magnified subregion near the origin.

$$
\dot{w}=\lambda_{A} w+\lambda_{D} w+\lambda_{R} w
$$

Denote $z_{A}=\tau \lambda_{A}$, etc. and $z=z_{A}+z_{D}+z_{R}$. The stability function of the factorized ROS2 scheme can then be written as

$$
R\left(z_{A}, z_{D}, z_{R}\right)=1+\frac{2 z}{\left(1-\gamma z_{D}\right)\left(1-\gamma z_{R}\right)}+\frac{\frac{1}{2} z^{2}-z}{\left(1-\gamma z_{D}\right)^{2}\left(1-\gamma z_{R}\right)^{2}} .
$$

Observe that due to the factorization there is no longer damping at infinity. For $z_{D}=z_{R}=0$ the stability function of the explicit trapezoidal rule is recovered.

We are interested in stability whenever $z_{D}<0$ is real and the explicit trapezoidal rule stability function satisfies $\left|1+z_{A}+\frac{1}{2} z_{A}^{2}\right| \leq 1$. This leads to a stability region for $z_{R}$ which we have determined numerically; see Figure 8 . The figure reveals $A(\alpha)$-stability for $z_{R}$. Recall that a method is said to be $A(\alpha)$-stable if the sector

$$
\{z:|\pi-\arg (z)|<\alpha, \operatorname{Re}(z)<0\}
$$

lies in the stability region. A careful inspection near the origin revealed that the angle $\alpha$ is very close to $39^{\circ}$. This angle probably is sufficiently large since eigenvalues with a large imaginary part do not seem to occur in atmospheric chemistry models. With a weaker condition on $z_{A}$ a larger angle will be found. In conclusion, with respect to stability for the linear model problem, the critical step size is equal to that of the explicit trapezoidal rule for the advection computation. This means that with respect to model problem stability, the factorized ROS2 scheme has the same stability characteristics as the two previous Strang-splitting schemes.

5.2. 3D results. We have compared the factorized ROS2 scheme (5.1) (5.2) to the Strang-splitting scheme (4.6) by repeating the 3D prototype model test with the 100 times larger diffusion coefficient $K$. Results are shown in Figure 7 in the same way as for the Strang-splitting scheme. As we anticipated, the huge error in $\mathrm{HO}_{2} \mathrm{NO}_{2}$ is now absent. On the other hand, for $\mathrm{O}_{3}$ the errors are comparable and for $\mathrm{HNO}_{3}$ the Strang-splitting solution is even slightly more accurate. Hence the factorized ROS2 scheme offers an improvement, but less than expected. In this respect it is emphasized that factorization as in (5.2) also introduces errors, as splitting does. By factorization 
we do use an approximation to the true Jacobian matrix $F^{\prime}\left(w_{n}\right)$. Approximating $F^{\prime}\left(w_{n}\right)$ in a Rosenbrock method leads to terms in the local truncation error different from the elementary differentials. The contribution of such new terms to the local truncation error is hard to predict, but it is most likely that they will increase the local error. With regard to CPU time the two schemes are comparable, but Strang-splitting is somewhat more convenient for economical memory use.

6. Source-splitting. During our investigations we have examined another alternative to Strang-splitting, which we call source-splitting. Source-splitting is advocated in $[12,13,22,24]$. The underlying idea is to treat the transport term as a constant source within the chemistry integration, so that a change of the solution values as happens in Strang-splitting is avoided. Similar to Strang-splitting, the idea can be implemented in different ways. Adopting it for ROS2 as chemistry scheme and the explicit trapezoidal rule as transport scheme for the system $\dot{w}=F_{T}(w)+F_{R}(w)$ yields

$$
\begin{aligned}
w_{n+1} & =w_{n}+\frac{3}{2} \tau k_{1}+\frac{1}{2} \tau k_{2}, \\
(I-\gamma \tau A) k_{1} & =F_{R}\left(w_{n}\right)+\tilde{F}_{T}, \\
(I-\gamma \tau A) k_{2} & =F_{R}\left(w_{n}+\tau k_{1}\right)+\tilde{F}_{T}-2 k_{1},
\end{aligned}
$$

where $A=F_{R}^{\prime}\left(w_{n}\right)$ and the source term $\tilde{F}_{T}$ is defined by

$$
\tilde{F}_{T}=\frac{1}{2} F_{T}\left(w_{n}\right)+\frac{1}{2} F_{T}\left(w_{n}+\tau F_{T}\left(w_{n}\right)\right)
$$

This scheme shares the implementational advantages of the related splitting scheme (4.2), but it is only of first order.

Treating transport as a source term requires a separate stability investigation. The stability function for the test model $\dot{w}=\lambda_{T} w+\lambda_{R} w$ reads

$$
R\left(z_{T}, z_{R}\right)=\frac{\left(1+z_{T}+\frac{1}{2} z_{T}^{2}\right)+(1-2 \gamma) z_{R}+\frac{1}{2}(1-4 \gamma) z_{R}\left(z_{T}+\frac{1}{2} z_{T}^{2}\right)}{\left(1-\gamma z_{R}\right)^{2}}
$$

We have found that with respect to stability the method is less stable than (4.2). Below we prove that $\left|R\left(z_{T}, z_{R}\right)\right| \leq 1$ whenever $z_{R} \leq 0$ is real and $\left|1+z_{T}+\frac{1}{2} z_{T}^{2}\right| \leq 1$, but that no positive angle $\alpha$ exists for which this stability function is $A(\alpha)$-stable with respect to $z_{R}$ uniformly for $z_{T}$ in the stability region of the explicit trapezoidal rule. In other words, unlike the Strang-splitting method (4.2), the source-splitting method does not simultaneously maintain the stability provided by the explicit trapezoidal rule for advection-diffusion and the L-stability of ROS2 for the chemistry.

LEMma 2. $\left|R\left(z_{T}, z_{R}\right)\right| \leq 1$ whenever $z_{R} \leq 0$ is real and $\left|1+z_{T}+\frac{1}{2} z_{T}^{2}\right| \leq 1$.

Proof. Put $1+z_{T}+\frac{1}{2} z_{T}^{2}=e^{i \phi}$. Then

$$
\begin{aligned}
R\left(z_{T}, z_{R}\right) & =\frac{e^{i \phi}+\left(e^{i \phi}-1\right)(1-4 \gamma) \frac{1}{2} z_{R}+(1-2 \gamma) z_{R}}{\left(1-\gamma z_{R}\right)^{2}} \\
& =\frac{e^{i \phi}\left(1+(1-4 \gamma) \frac{1}{2} z_{R}\right)+\frac{1}{2} z_{R}}{\left(1-\gamma z_{R}\right)^{2}}
\end{aligned}
$$

Suppose $z_{R}=-t, t \geq 0$ and write $\alpha=1-\frac{1}{2}(1-4 \gamma) t$. Then

$$
\left|R\left(z_{T}, z_{R}\right)\right|^{2}=\frac{\left(\alpha \cos (\phi)-\frac{1}{2} t\right)^{2}+\alpha^{2} \sin ^{2}(\phi)}{(1+\gamma t)^{4}} .
$$


A straightforward calculation now shows that $\left|R\left(z_{T}, z_{R}\right)\right| \leq 1$ for all $t \geq 0$ and all $\phi$. The result of the lemma then follows from the maximum modulus theorem.

LEMMA 3. No positive angle $\alpha$ exists for which the source-splitting stability function (6.3) is $A(\alpha)$-stable with respect to $z_{R}$ uniformly for $z_{T}$ in the stability region of the explicit trapezoidal rule.

Proof. Letting $z_{R}$ sufficiently small in (6.4) yields

$$
R\left(z_{R}, z_{T}\right)=e^{i \phi}+\frac{1}{2} z_{R}\left(1+e^{i \phi}\right)+O\left(z_{R}^{2}\right) .
$$

Substitution of $z_{R}=a+i b$ with $a \leq 0$ and $e^{i \phi}=\cos \phi+i \sin \phi$ yields

$$
\left|R\left(z_{R}, z_{T}\right)\right|^{2}=1+a(1+\cos \phi)+b \sin \phi+\frac{1}{2}\left(a^{2}+b^{2}\right)(1+\cos \phi)+O\left(z_{R}^{2}\right) .
$$

Hence, in first approximation we can write

$$
\left|R\left(z_{R}, z_{T}\right)\right|^{2}=1+a(1+\cos \phi)+b \sin \phi .
$$

Choose $\phi=\pi+\epsilon$ with $\epsilon>0$ and also arbitrarily small. Then, again in first approximation,

$$
\left|R\left(z_{R}, z_{T}\right)\right|^{2}=1-b \epsilon+\frac{1}{2} a \epsilon^{2}+O\left(\epsilon^{3}\right) .
$$

We see that $1-b \epsilon+\frac{1}{2} a \epsilon^{2}>1$ if $b<\frac{1}{2} a \epsilon$. Hence for any sufficiently small real part $a$, an arbitrarily small imaginary part $b$ exists such that $\left|R\left(z_{R}, z_{T}\right)\right|>1$, showing that no positive angle $\alpha$ exists defining $A(\alpha)$-stability.

Numerical experiments with method (6.1)-(6.2) applied to the 3D prototype model using $K_{\max }=30$ revealed instability. In a similar vein we have studied a source-splitting counterpart of the Strang-splitting method (4.6). For this alternative source-splitting method, using the implicit-explicit transport solver (4.5) instead of the explicit trapezoidal rule, essentially the same restrictive linear stability results do hold as for (6.1)-(6.2). Surprisingly, applied to the 3D problem with the 100 times larger vertical diffusion coefficient, the alternative method based on (4.5) was stable and in fact equally accurate as the approximate factorization ROS2 method (5.1)(5.2). As yet our findings on source-splitting are therefore inconclusive. Apparently, the precise meaning of the lack of $\mathrm{A}(\alpha)$-stability for $z_{R}$ must be reconsidered, as well as the use of the implicit-explicit transport solver (4.5) versus the explicit trapezoidal rule.

7. Main conclusion. Until now Strang-type operator splitting seemed the method of choice for time stepping in global air quality modeling in spite of the occurrence of splitting errors. We have found it reliable and it provides flexibility, both for model and code development. Within splitting, one of the most time-consuming computations is the stiff chemistry integration. Due to artificial transients introduced at the beginning of split intervals, a highly stable solver is required. This solver should be able to use large time steps in the order of minutes, being far greater than the smallest time constants, which are in the order of milliseconds and even smaller. In addition, for convenient code design on vector/parallel and massively parallel computers, this solver should be able to cope with such large step sizes equally distributed over the whole space grid, or large parts thereof, under widely inhomogeneous spatial and temporal conditions. For three different sets of chemical reactions we have demonstrated that the sparse Rosenbrock solver ROS2 is an excellent candidate. An open question still is how ROS2 will perform under even more difficult real atmospheric and meteorological conditions. Likewise, it is of interest to examine the role of the splitting 
error under such conditions. Finally, ROS2 can of course also prove useful for use in related reactive-flow computations where Strang-splitting is used.

\section{REFERENCES}

[1] I. Ahmad And M. Berzins, An algorithm for ODEs from atmospheric dispersion problems, Appl. Numer. Math., 25 (1997), pp. 137-150.

[2] R. M. BeAm And R. F. Warming, An implicit finite-difference algorithm for hyperbolic systems in conservation-law form, J. Comput. Phys. 22 (1976), pp. 87-110.

[3] R. F. WARming AND R. M. BEam, An extension of A-stability to alternating direction methods, BIT 19 (1979), pp. 395-417.

[4] V. Damian-IORDaChe ANd A. SAndu, KPP-A Symbolic Preprocessor for Chemistry KineticsUser's Guide, Department of Mathematics, University of Iowa, Iowa City, IA, 1995.

[5] K. DekKer and J. G. Verwer, Stability of Runge-Kutta Methods for Stiff Nonlinear Differential Equations, Elsevier-North Holland, Amsterdam, 1984.

[6] H. Elbern, Parallelization and load balancing of a comprehensive atmospheric chemistry transport model, Atmos. Environ., 31 (1997), pp. 3561-3574.

[7] E. Hairer And G. WANNer, Solving Ordinary Differential Equations II. Stiff and DifferentialAlgebraic Problems, 2nd ed., Springer-Verlag, Berlin, 1996.

[8] O. Hertel, R. Berkowicz, J. Christensen, AND $\varnothing$. Hov, Test of two numerical schemes for use in atmospheric transport-chemistry models, Atmos. Environ., 27 (1993), pp. 2591-2611.

[9] W. Hundsdorfer, B. Koren, M. van LOON, AND J. G. Verwer, A positive finite-difference advection scheme, J. Comput. Phys., 117 (1995), pp. 35-46.

[10] M. Z. JaCOBSON AND R. P. TURCo, SMVGEAR: A sparse-matrix, vectorized gear code for atmospheric models, Atmos. Environ., 28 (1994), pp. 273-284.

[11] L. O. JAY, A. SAndu, F. A. Potra, AND G. R. CARmichael, Improved quasi-steady-stateapproximation methods for atmospheric chemistry integration, SIAM J. Sci. Comput., 18 (1997), pp. 182-202.

[12] CHR. KessLER, Entwicklung eines Effizienten Lösungsverfahrens zur Modellmäßigen Beschreibung der Ausbreitung und Chemischen Umwandlung Reaktiver Luftschadstoffe, Ph.D. thesis, Fakultät für Maschinenbau der Universität Karlsruhe, Verlag Shaker, Aachen, 1995.

[13] O. KNOTH AND R. WOLKE, A comparison of fast chemical kinetic solvers in a simple vertical diffusion model, in Proceedings 20th International Meeting on Air Pollution Modelling and its Applications, Valencia, Spain, 1993.

[14] J. MatThiJsen, private communication, Laboratoire d'Aerologie OMP, Toulouse, France, 1995.

[15] G. J. MCRAE, W. R. Goodin, ANd J. H. Seinfeld, Numerical solution of the atmospheric diffusion equation for chemically reacting flows, J. Comput. Phys., 45 (1982), pp. 1-42.

[16] A. Sandu, F. A. Potra, G. R. Carmichael, and V. Damian, Efficient implementation of fully implicit methods for atmospheric chemical kinetics, J. Comput. Phys., 129 (1996), pp. 101-110.

[17] A. Sandu, J. G. Verwer, J. G. Blom, E. J. Spee, and G. R. Carmichael, Benchmarking stiff $O D E$ solvers for atmospheric chemistry problems II: Rosenbrock solvers, Atmos. Environ., 31 (1997), pp. 3459-3472.

[18] A. Sandu, J. G. Verwer, M. van Loon, G. R. Carmichael, F. A. Potra, D. Dabdub, and J. H. SEINFELD, Benchmarking stiff ODE solvers for atmospheric chemistry problems I: Implicit versus explicit, Atmos. Environ., 31 (1997), pp. 3151-3166.

[19] A. Sandu, Numerical Aspects of Air Quality Modeling, Ph.D. thesis, University of Iowa, Iowa City, IA, 1997.

[20] P. K. Smolarkiewicz and P. J. Rasch, Monotone advection on the sphere: An Eulerian versus semi-Lagrangian approach, J. Atmos. Sci., 48 (1991), pp. 793-810.

[21] E. J. Spee, P. M. DE Zeeuw, J. G. Verwer, J. G. Blom, And W. Hundsdorfer, A Numerical Study for Global Atmospheric Transport-Chemistry Problems, Revision of CWI Report MAS-R9702, CWI, Amsterdam, 1997.

[22] B. Sportisse, A. Jaubertie, AND P. Plion, Reducing mechanism in chemical kinetics for the simulation of reactive transport: An application to air pollution modeling, in Proceedings Saint-Venant Symposium, INRIA, Paris, 1997.

[23] G. StRAnG, On the construction and comparison of difference schemes, SIAM J. Numer. Anal., 5 (1968), pp. 506-517.

[24] P. Sun, A pseudo-non-time-splitting method in air quality modeling, J. Comput. Phys., 127 (1996), pp. 152-157.

[25] P. J. VAN DER HOUWEN, B. P. SOMMEIJER, AND J. KOK, The iterative solution of fully implicit 
discretizations of three-dimensional transport models, Appl. Numer. Math., 25 (1997), pp. 243-256.

[26] J. G. Verwer, S-stability properties for generalized Runge-Kutta methods. Numer. Math., 27 (1977), pp. 359-370

[27] J. G. VERWER, Gauss-Seidel iteration for stiff ODEs from chemical kinetics, SIAM J. Sci. Comput., 15 (1994), pp. 1243-1250.

[28] J. G. VERWER AND J. G. BLOM, On the coupled solution of diffusion and chemistry in air pollution models, in Proceedings of the Third International Congress on Industrial and Applied Mathematics (ICIAM/GAMM 95), ZAMM, Issue 4: Applied Sciences, Especially Mechanics, E. Kreuzer and O. Mahrenholtz, eds., Akademie Verlag, Berlin, 1996, pp. 454457.

[29] J. G. Verwer, J. G. Blom, M. van Loon, and E. J. Spee, A comparison of stiff ODE solvers for atmospheric chemistry problems, Atmos. Environ., 30 (1995), pp. 49-58.

[30] J. G. VERWER AND D. SIMPSON, Explicit methods for stiff ODEs from atmospheric chemistry, Appl. Numer. Math, 18 (1995), pp. 413-430.

[31] P. WARnECK, Chemistry of the Natural Atmosphere, Academic Press, San Diego, CA, 1988.

[32] Z. ZLATev, Computer Treatment of Large Air Pollution Models, Kluwer Academic Publishers Norwell, MA, 1995. 